\title{
Los anticonceptivos orales con drospirenona se asociaron a un incremento en el riesgo de tromboembolismo venoso
}

\author{
Drospirenone contining oral contraceptives were associated with an increased risk of venous thrombosis
}

\section{Objetivo}

Evaluar el riesgo de tromboembolismo venoso (TEV), infarto de miocardio (IM), y accidentes cerebrovasculares (ACV) en mujeres usuarias de anticonceptivos orales (ACO) que contienen drospirenona.

\section{Fuentes de datos y selección de estudios}

CINAHL, Cochrane Library, Dissertation Abstracts, EMBASE, HealthStar, MEDLINE, y Science Citation Index (desde sus inicios y hasta noviembre 2012). Se incluyeron todos los reportes de casos, serie de casos y estudios observacionales que evaluaran el riesgo de trombosis arterial y venosa en usuarias de ACO con drospirenona. La búsqueda se limitó a estudios realizados en población adulta femenina, publicados en inglés o francés.

\section{Extracción de datos}

La recopilación y análisis de datos fue realizada de manera independiente por dos revisores. Fueron incluidos un total de 22 estudios (seis reportes de casos, tres series de casos y 13 estudios comparativos). Los reportes y series de casos contenían un total de 32 casos de eventos trombóticos asociados a drospirenona. Veinte de estas 32 mujeres tenían al menos un factor de riesgo conocido para enfermedad tromboembólica. La edad media fue de 33,5 años (rango, 17 a 50 años) y la duración media del uso antes del evento trombótico fue de 150,5 días (rango, 15 a 2.557 días).

Del total de 13 estudios comparativos hallados nueve eran cohortes, y los restantes cuatro, casos y controles. No se identificó ningún ensayo clínico aleatorizado. La duración del seguimiento varió desde 12 hasta 180 meses.
Wu C y col. BJOG. 2013;120(7):801-10.

Resultados Principales

Se resumen en las tablas 1 y 2 . Dos estudios analizaron el riesgo con usuarias de otros ACO, los resultados no fueron concluyentes. Los efectos relacionados con trombosis arterial de los ACO que contienen drospirenona tampoco fueron concluyentes.

Tabla 1. Riesgo de tromboembolismo venoso en usuarias de anticonceptivos orales con drospirerona vs. levonorgestrel (ocho estudios).

\begin{tabular}{|c|c|c}
\hline Drospirenona & Levonorgestrel & \multirow{2}{*}{ RR } \\
\hline \multicolumn{2}{|c|}{ (Tasa de incidencia cada 100.000 mujeres-año) } & \\
\hline 23,0 a 136,7 & 6,64 a 92,1 & 1,0 a 3,3 \\
\hline
\end{tabular}

Tabla 2. Riesgo de tromboembolismo venoso en usuarias de anticonceptivos orales con drospirenona vs. no usuarias de anticonceptivos orales (tres estudios).

\begin{tabular}{c|c|c} 
Drospirenona & No usuarias & \multirow{2}{*}{ RR } \\
\cline { 1 - 1 } \multicolumn{2}{c|}{ (Tasa de incidencia cada 100.000 mujeres-año) } & \\
\hline 78,3 a 93 & No usuarias & 4,0 a 6,3 \\
\hline
\end{tabular}

\section{Conclusiones}

Esta revisión sistemática sugiere que el uso de ACO con drospirenona se asocia con un mayor riesgo de TEV en comparación con usuarias de ACO con levonorgestrel o aquellas que no utilizan ACO.

Fuente de financiamiento: Canadian Institutes of Health Research.

\section{Comentario}

La evidencia hasta la fecha sugiere que los ACO que contienen drospirenona pueden aumentar el riesgo de TEV. Esta tendencia es consistente con las conclusiones elaboradas a partir de estudios previos que investigan el riesgo trombótico con el uso de progestagenos de segunda y tercera generación'. En el 2011, la Agencia reguladora de Medicinas y Productos Sanitarios del Reino Unido (MHRA), la FDA de EE.UU. y Canadá Salud realizaron informes de alerta que concluían que los ACO con dropsirenona podrían asociarse con 1,5 a 3 veces mayor riesgo de TEV. Estos resultados son compatibles con los resultados de esta revisión sistemática ${ }^{2}$. Los efectos de la drospirenona sobre el riesgo de trombosis arterial siguen sin estar claros, ya que algunos estudios sugirieron un efecto protector mientras que otros sugieren una duplicación del riesgo. Si bien la evidencia de esta revisión es fuerte, cuenta con algunas limitaciones metodológicas. Dada la heterogeneidad de los estudios, definiciones de usuarios y medidas de efecto, no se pudieron agrupar los datos para obtener una única estimación global y de síntesis (meta-análisis). Por otra parte, la dosis de estrógenos no fue especificada en varios de los estudios incluidos. Además, basándose en la propiedad anti-mineralocorticoide y anti-androgénica de la drospirenona, los ACO que con- tienen este progestina podrían haber sido preferentemente recetados a mujeres con un mayor riesgo de TEV y trombosis arterial ${ }^{3}$. Además, esta revisión sistemática se limitó a estudios publicados en inglés o francés. Es importante destacar que, si bien los ACO que contienen drospirenona parecen aumentar el riesgo de TEV, y tienen efectos poco claro en el riesgo de trombosis arterial, el riesgo absoluto de trombosis cuando se utilizan estos agentes sigue siendo bajo ${ }^{4}$, aún menor que el riesgo de eventos trombóticos durante el embarazo o posparto (se estima que la incidencia de TEV puede ser de 9 a 10 casos por cada 10.000 mujeres-año para usuarias de ACO, frente a 5 a 10 por 10.000 para las no usuarias, pero de aproximadamente 29 por 10.000 durante el embarazo y de 300 a 400 por cada 10.000 durante el posparto ${ }^{5}$.

\section{Conclusiones del comentador}

Las mujeres deben comprender los riesgos y beneficios asociados con el uso de estos agentes para permitir una toma de decisión informada ya que la eficacia de los anticonceptivos orales depende del cumplimiento, por lo tanto de la satisfacción con el método anticonceptivo elegido.

\section{Nadia Silvina Musarella [ Servicio de Medicina Familiar y Comunitaria del Hospital Italiano de Buenos Aires. nadia.musarella@ hospitalitaliano.org.ar ]}

Musarella N. Los anticonceptivos orales con drospirenona se asociaron a un incremento en el riesgo de tromboembolismo venoso. Evid Act Pract Ambul. 2015;18(3)82. Jul-Sep. Comentado de: Wu, CQ, y col. Drospirenone-containing oral contraceptive pills and the risk of venous and arterial thrombosis: a systematic review. BJOG. 2013;120(7):801-10. PMID: 23530659.

\section{Referencias}

1. Gronich $\mathrm{N}$, y col. Higher risk of venous thrombosis associated with drospirenone-containing oral contraceptives: a population-based cohort study. CMAJ 2011;183:E1319-25.

2. US. Food and Drug Administration. FDA Drug Safety Communication: Updated information about the risk of blood clots in women taking birth control pills containing drospirenone. Maryland, EE.UU; 2012. Disponible en URL: http://www.fda.gov/Drugs/DrugSafety/ucm299305 (último acceso, junio 2015).

3. Heinemann LA, Dinger J. Safety of a new oral contraceptive containing drospirenone. Drug Saf 2004:27:1001-18.

4. Lidegaard O, y col. Thrombotic stroke and myocardial infarction with hormonal contraception. N Engl J Med 2012;366:2257-66.

5. Faculty of Sexual \& Reproductive Healthcare. Combined hormonal contraception. England, 2011

Disponible en URL: http://www.fsrh.org/pdfs/CEUGuidanceCombinedHormonalContraception.pdf (último acceso, junio 2015). 